\title{
Diversidade das assembléias de peixes nas quatro unidades geográficas do rio Paraíba do Sul
}

\author{
Tatiana P. Teixeira ${ }^{1,2}$, Benjamin C. T. Pinto ${ }^{1,3}$, Bianca de Freitas Terra ${ }^{1,2}$, Eduardo O. Estiliano ${ }^{1,4}$, \\ Daniel Gracia ${ }^{1} \&$ Francisco G. Araújo ${ }^{1}$
}

1. Universidade Federal Rural do Rio de Janeiro, Laboratório de Ecologia de Peixes, IB/DBA, km 47, Antiga Rodovia Rio São Paulo,
23.851-970 Seropédica, RJ. (gerson @ufrrj.br)
2. Bolsista PIBIC/CNPq.
3. Programa de Pós-Graduação em Biologia Animal, Instituto de Biologia, Universidade Federal Rural do Rio de Janeiro.
4. Programa de Pós-Graduação em Ciências Ambientais e Florestais, Instituto de Floresta, Universidade Federal Rural do Rio de Janeiro.

ABSTRACT. Diversity of fish assemblages in the four geographic units of the Paraíba do Sul river. Diversity patterns of fish assemblages along the four geographic units (upper, middle-upper, middle-lower and lower reaches) of the Paraíba do Sul river were studied aiming to assess spatial (geographic units) and seasonal (wet/dry periods) variations. Twenty-five sites were sampled between December 2002 and March 2003 (summer/wet) and, August to November 2003 (winter/dry). Fish were caught by a standardized effort, using gill nets, cast nets and mesh trays. A total of 81 species were recorded comprised in 9 orders, 29 families and 55 genera. Characiformes showed the highest number of species (28) followed by Siluriformes (23). Perciformes, mainly Tilapia rendalli and Geophagus brasiliensis, and Cyprinodontiformes, mainly Poecilia vivipara and Poecilia reticulata, were the most numerically abundant groups, while Siluriformes, mainly Hypostomus luetkeni, and Perciformes, mainly Geophagus brasiliensis showed the highest biomass. Poecilia vivipara was recorded only in dry period. Spatially, Hoplosternum littorale predominated in the middle-upper reaches, Pimelodus fur, Hypostomus luetkeni, Glanidium albescens in the middle-lower, and Loricariichtys spixii and Prochilodus lineatus, in the lower reach. Number of species and Margalef's richness showed an increase from the upper to the lower river reaches, mainly during the wet period. Species widely distributed along all over the river extent (G. brasiliensis, Oligosarcus hepsetus e P. reticulata) are opportunistic and can use the available resources despite poor environmental conditions, reflecting the state alteration of the river. Differentiation on the physiography throughout the longitudinal extent of the basin does not match shifts in fish assemblages, suggesting that factors associated to habitat alterations could be structuring fish community at local scale.

KEYWORDS. Fish assemblages, spatial patterns, fish distribution, ichthyofauna, neotropical rivers.

RESUMO. Padrões na diversidade de peixes ao longo de quatro unidades geográficas (superior, médio-superior, médio-inferior e inferior) no rio Paraíba do Sul foram estudados com a finalidade de avaliar tendências de variações espaciais (unidades geográficas) e temporais (períodos de cheia e seca). Vinte e cinco locais foram amostrados entre Dezembro de 2002 e Março de 2003 (verão/cheia) e, entre Agosto e Novembro de 2003 (inverno/seca). Os peixes foram capturados com um esforço padronizado, utilizando redes de espera, tarrafas e peneiras. Um total de 81 espécies foram registradas compreendendo 9 ordens, 29 famílias e 55 gêneros. Characiformes apresentaram maior número de espécies (28) seguido de Siluriformes (23). Perciformes, principalmente Tilapia rendalli e Geophagus brasiliensis, e Cyprinodontiformes com destaque para Poecilia vivipara e Poecilia reticulata, foram os grupos numericamente mais abundantes, enquanto Siluriformes, principalmente Hypostomus luetkeni, e Perciformes com Geophagus brasiliensis apresentaram maior contribuição em biomassa. Poecilia vivipara foi encontrada apenas no período de seca. Espacialmente, Hoplosternum littorale predominou no trecho médio-superior, Pimelodus fur, Hypostomus luetkeni, Glanidium albescens no trecho médio-inferior, e Loricariichtys spixii e Prochilodus lineatus no trecho inferior. O número de espécies e a riqueza de Margalef apresentaram um aumento do trecho superior para o inferior, principalmente durante o período de cheia. Espécies que apresentaram ampla distribuição ao longo do rio (G. brasiliensis, Oligosarcus hepsetus e $P$. reticulata) são consideradas oportunistas por se aproveitarem dos recursos disponíveis em ambientes pobres, refletindo o estado de alterações do rio. Diferenciações na fisiografia ao longo da extensão longitudinal da bacia não coincidiram com mudanças nas assembléias de peixes, sugerindo que fatores associados a alterações de hábitats poderiam estar estruturando a comunidade de peixes a nível local.

PALAVRAS-CHAVE. Assembléias de peixes, padrões espaciais, distribuição de peixes, ictiofauna, rios neotropicais.

O rio Paraíba do Sul é um dos mais utilizados sistemas lóticos do Brasil, por situar-se entre os maiores centros urbano-industriais do país, e tem sofrido alterações de origem antrópica ao longo dos últimos quatro séculos. A intensa urbanização e industrialização são os maiores causadores dos efeitos negativos na qualidade da água e dos hábitats, onde a precariedade do saneamento básico (esgotamento sanitário e resíduos sólidos), o uso de efluentes para escoamento de resíduos industriais, o desmatamento da vegetação ripariana e o uso indiscriminado de insumos agrícolas resultam na baixa qualidade da água (AMORIN et al., 1983). A determinação da biodiversidade, especialmente das assembléias de peixes e dos seus padrões de variação espaciais e temporais, é de grande relevância para avaliar a qualidade ambiental, uma vez que os peixes ocupam variadas posições na teia trófica. $\mathrm{O}$ monitoramento biológico da ictiofauna em rios também é essencial para identificar as respostas do ambiente aos impactos causados pela ação antrópica, além de fornecer subsídios para regulamentação dos usos dos recursos hídricos, possibilitando o desenvolvimento de alternativas para minimizar a degradação dos rios.

Os hábitats de um rio desempenham importante papel na integridade ecológica, com os peixes utilizando as dimensões espaciais e temporais do nicho de várias maneiras. Algumas espécies são altamente dependentes da integridade dos hábitats, pois são residentes com todo 
o ciclo de vida ocorrendo em uma área limitada, apresentando movimentos restritos para desova e alimentação; outras espécies são migratórias e utilizam diferentes partes do curso do rio durante o ciclo de vida (Freire \& Agostinho, 2000). Algumas espécies ocorrem principalmente no canal principal e outras são adaptadas aos pequenos tributários. Neste trabalho procurou-se abordar a distribuição espacial e temporal das espécies que ocupam o canal principal e os maiores tributários do rio Paraíba do Sul.

É esperado que a comunidade de peixes se modifique conforme os trechos do rio, como resultado dos processos evolutivos e históricos de adaptações peculiares de cada espécie, modulados por influências ambientais, condições de hábitats e alteradas por influências de origem antrópica (MARTIN-SMITH, 1998; MEAdOR \& Goldestein, 2003). De acordo com a teoria do Rio Contínuo (VANNOTE et al., 1980), as variáveis físicas de um rio natural apresentam gradiente contínuo de montante para jusante, com as comunidades biológicas se ajustando, através da substituição de espécies, no sentido de usar com maior eficiência a energia. Trechos de rios cujo contenuo longitudinal é artificialmente fragmentado, mas que são ricos em estruturas físicas, podem suportar populações viáveis por fornecerem suficiente alimento e áreas de reprodução (JUNGWIRTH et al., 2000). Alterações dos hábitats, tais como, canalização das margens, destruição da vegetação ripária ou barramentos, além de introduções de espécies não-nativas e a deterioração da qualidade da água, exercem uma profunda e negativa influência no ecossistema, podendo causar variações na distribuição espaço-temporal na comunidade de peixes (WAITE \& CARPENTER, 2000). Tais modificações precisam, portanto, ser avaliadas e monitoradas, diagnosticando os possíveis padrões estruturadores destas assembléias.

A variação sazonal no nível de fluxo da água é outro fator estruturador das comunidades de peixe dos rios (Wootton, 1992). Nos trechos de planícies de inundações, a diversidade e densidade de peixes são determinadas pelo regime de fluxos; a inundação aumenta a homogeneização das características limnológicas do ambiente (THomAz et al., 1997) e os ambientes passam a responder aos fatores regionais independentemente, aumentando a heterogeneidade da fauna na medida que os microhábitats são isolados no período de seca.

$\mathrm{O}$ presente trabalho teve por objetivo descrever os padrões espaço-temporais da diversidade da ictiofauna do rio Paraíba do Sul ao longo das quatro unidades geográficas do trecho longitudinal e dos períodos cheia/seca.

\section{MATERIAL E MÉTODOS}

A bacia do rio Paraíba do Sul encontra-se compreendida entre os paralelos $20^{\circ} 26^{\prime}$ e $23^{\circ} 38^{\prime}$ sul e os meridianos $41^{\circ} 00^{\prime}$ e $46^{\circ} 30^{\prime}$ oeste. Ao norte seu divisor de águas se faz entre os rios Grande (bacia do Paraná) e Doce (sistema do leste brasileiro) por intermédio da Serra da Mantiqueira; ao Sul, a Serra do Mar separa esta bacia de diversos pequenos rios que fluem diretamente para o Oceano Atlântico.
Quatro unidades geográficas foram definidas por Ab'Saber \& Bernardes (1958) apud BIZERRIL (1999) para o rio Paraíba do Sul, com base em características topográficas e ambientais: trecho superior, médiosuperior, médio-inferior e inferior. O trecho superior, por situar-se em região de maior altitude, onde não se inserem grandes centros urbanos ou industriais, é conseqüentemente, o menos alterado. Os trechos mais à jusante, todos drenam áreas onde estão inseridos grandes centros urbano-industriais, como é o caso do trecho médio superior, onde se localiza o vale Paulista do Paraíba, destacando-se os municípios de São José dos Campos, Taubaté e Cachoeira Paulista; o trecho médio-inferior, com o complexo industrial de Resende a Volta Redonda, e o trecho inferior, com destaque para monocultura de canade-açúcar e indústrias associadas se destacam com atividades de grande importância.

Os maiores fluxos correspondem ao período do verão (chuvoso) e os menores ao inverno (seco). O período chuvoso apresenta certa regularidade, iniciandose geralmente em novembro e prolongando-se até o início de outono. Os máximos ocorrem em janeiro e, ocasionalmente, em março. Em um dos locais amostrados no trecho médio-inferior (Piraí) os valores históricos de pluviosidade encontrados no verão e inverno foram de $599 \mathrm{~mm}$ e $151 \mathrm{~mm}$ respectivamente (BARBIERE \& KRONEMBERG, 1994). No verão, as maiores pluviosidades carreiam para o rio maior quantidade de material alóctone, aumentando a disponibilidade de alimento; em áreas de intensa atividade humana, perturbações podem ser causadas por picos de pluviosidade, devido ao aumento do material em suspensão.

Foi estudada toda a extensão do rio Paraíba do Sul, desde as nascentes formadas pela confluência dos rios Paraitinga e Paraibuna na serra da Bocaina no Estado de São Paulo, até as proximidades do estuário, no município de São João da Barra no norte do Estado do Rio de Janeiro. Um total de 25 locais foi amostrado (Fig. 1; Tab. I), sendo 23 deles no período de cheia, e 18 no período de seca, compreendendo além do canal principal do rio Paraíba do Sul, os dois formadores deste rio (Rios Paraibuna e rio Paraitinga) e os tributários de grande porte: Paraibuna (de Minas Gerais), Preto, Piabanha, Pomba, Muriaé e Grande. O número de amostras variou entre os trechos devido às diferentes extensões dos mesmos e limitações de acessibilidade a locais que permitissem a utilização de um esforço padronizado. Foram investigados os dois períodos de maior diferenciação na pluviosidade e, por conseqüência, do fluxo da água: período chuvoso, entre novembro de 2002 e março de 2003; e período de seca, entre agosto e novembro de 2003. A maioria dos locais foi amostrada em ambos os períodos, outros locais foram substituídos e eliminados por dificultarem a padronização do esforço amostral.

O gradiente espacial foi avaliado através de amostragens em locais distribuídos ao longo do trecho longitudinal do rio, tendo-se utilizado as unidades geográficas estabelecidas por Ab'Saber \& Bernardes (1958) apud BIZERRIL (1999): 1) trecho superior (locais 1 a 5), com $280 \mathrm{~km}$ a partir da nascente, limitado à jusante pela cidade de Guararema; 2) trecho médio-superior (locais 


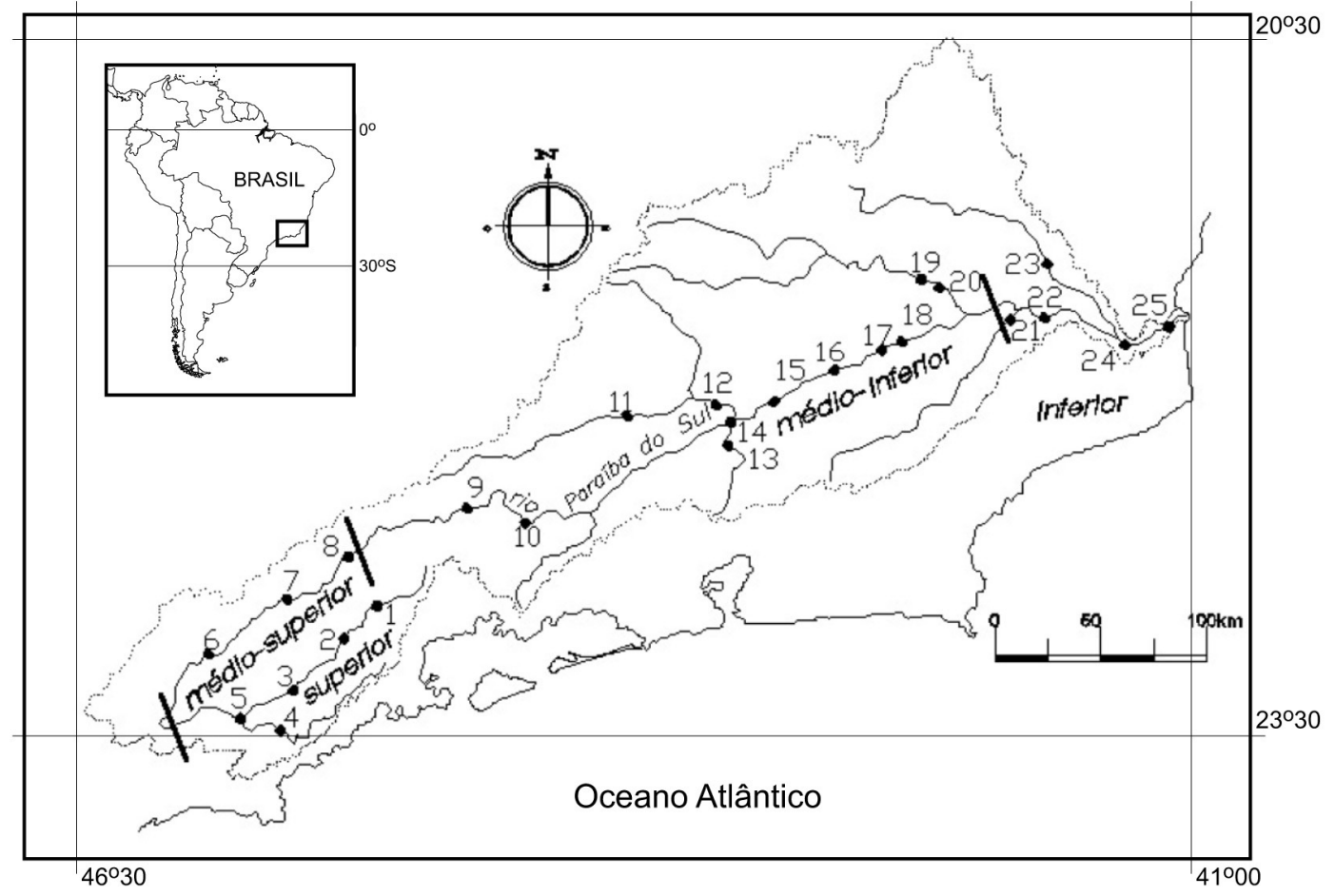

Fig. 1. Área da bacia hidrográfica do rio Paraíba do Sul, com indicação dos 25 locais de coleta (Vide Tab. I).

Tabela I. Locais de amostragem de peixes em quatro unidades geográficas e por período do ano (cheia / seca) no rio Paraíba do Sul, 2002/ 2003.

\begin{tabular}{|c|c|c|c|c|c|c|}
\hline & Local & Período & Município & Rio & Latitude & Longitude \\
\hline \multirow[t]{6}{*}{ Superior } & 1 & Cheia & Campos Novos de Cunha, SP & Paraitinga & $22^{\circ} 53,328^{\prime}$ & $44^{\circ} 54,761^{\prime}$ \\
\hline & 2 & Cheia & Campos Novos de Cunha, SP & Mato Dentro & $22^{\circ} 54,959^{\prime}$ & $44^{\circ} 49,050^{\prime}$ \\
\hline & 3 & Seca & Natividade da Serra, SP & Paraitinga & $23^{\circ} 23,160^{\prime}$ & $45^{\circ} 27,14^{\prime}$ \\
\hline & 4 & Seca & São Luís do Paraitinga, SP & Paraitinga & $23^{\circ} 12,705^{\prime}$ & $45^{\circ} 17,558^{\prime}$ \\
\hline & 5 & Cheia/seca & Paraibuna, SP & Encontro dos rios & $23^{\circ} 21,903^{\prime}$ & $45^{\circ} 40,013^{\prime}$ \\
\hline & & & & Paraibuna e Paraitinga & & \\
\hline \multirow[t]{3}{*}{ Médio-Superior } & 6 & Cheia/seca & São José dos Campos, SP & Paraíba do Sul & $23^{\circ} 10,813^{\prime}$ & $45^{\circ} 54,813^{\prime}$ \\
\hline & 7 & Cheia & Guaratinguetá, SP & Piagui & $22^{\circ} 46,273^{\prime}$ & $45^{\circ} 10,604^{\prime}$ \\
\hline & 8 & Cheia/seca & Cachoeira Paulista, SP & Paraíba do Sul & $22^{\circ} 30,308^{\prime}$ & $44^{\circ} 05,712^{\prime}$ \\
\hline \multirow[t]{10}{*}{ Médio-Inferior } & 9 & Cheia/seca & Barra Mansa, RJ & Paraíba do Sul & $22^{\circ} 28,251^{\prime}$ & $44^{\circ} 14,597^{\prime}$ \\
\hline & 10 & Cheia/seca & Volta Redonda, RJ & Paraíba do Sul & $22^{\circ} 30,308^{\prime}$ & $44^{\circ} 05,712^{\prime}$ \\
\hline & 11 & Cheia/seca & Rio das Flores, RJ & Preto & $22^{\circ} 03,454^{\prime}$ & $43^{\circ} 26,421^{\prime}$ \\
\hline & 12 & Cheia/seca & Rio das Flores, RJ & Paraibuna (mineiro) & $22^{\circ} 06,782^{\prime}$ & $43^{\circ} 27,200^{\prime}$ \\
\hline & 13 & Cheia & Petrópolis, RJ & Piabanha & $22^{\circ} 17,439^{\prime}$ & $43^{\circ} 07,584^{\prime}$ \\
\hline & 14 & Cheia/seca & Três Rios, RJ & Paraíba do Sul & $22^{\circ} 06,193^{\prime}$ & $43^{\circ} 07,514^{\prime}$ \\
\hline & 15 & Cheia & Três Rios, RJ & Paraíba do Sul & $23^{\circ} 06,578^{\prime}$ & $43^{\circ} 09,047^{\prime}$ \\
\hline & 16 & Cheia/seca & Anta, RJ & Paraíba do Sul & $22^{\circ} 01,923^{\prime}$ & $43^{\circ} 00,097^{\prime}$ \\
\hline & 17 & Cheia/seca & Sapucaia-RJ & Paraíba do Sul & $21^{\circ} 55,347^{\prime}$ & $42^{\circ} 46,389^{\prime}$ \\
\hline & 18 & Cheia & Além Paraíba, MG & Paraíba do Sul & $21^{\circ} 50,075^{\prime}$ & $42^{\circ} 38,216^{\prime}$ \\
\hline \multirow[t]{7}{*}{ Inferior } & 19 & Cheia & Santo Antônio de Pádua, RJ & Pomba & $21^{\circ} 30,002^{\prime}$ & $42^{\circ} 13,664^{\prime}$ \\
\hline & 20 & Cheia/seca & Santo Antônio de Pádua, RJ & Pomba & $21^{\circ} 32,285^{\prime}$ & $42^{\circ} 09,524^{\prime}$ \\
\hline & 21 & Cheia/seca & São Fidelis, RJ & Paraíba do Sul & $21^{\circ} 38,714^{\prime}$ & $41^{\circ} 44,745^{\prime}$ \\
\hline & 22 & Cheia/seca & São Fidelis, RJ & Dois Rios & $21^{\circ} 37,714^{\prime}$ & $41^{\circ} 49,620^{\prime}$ \\
\hline & 23 & Cheia/seca & Campos dos Goytacases, RJ & Paraíba do Sul & $21^{\circ} 43,470^{\prime}$ & $41^{\circ} 21,504^{\prime}$ \\
\hline & 24 & Cheia/seca & Italva, RJ & Muriaé & $21^{\circ} 25,075^{\prime}$ & $41^{\circ} 41,663^{\prime}$ \\
\hline & 25 & Cheia/seca & São João da Barra, RJ & Paraíba do Sul & $21^{\circ} 43,015^{\prime}$ & $41^{\circ} 10,205^{\prime}$ \\
\hline
\end{tabular}


6 a 8), compreendendo um trecho de $300 \mathrm{~km}$, tendo como limite à jusante a cidade de Cachoeira Paulista; 3) trecho médio-inferior (locais 9 a 18), com $430 \mathrm{~km}$ limitado pela cidade de São Fidélis, e 4) trecho inferior (locais 19 a 25), com $90 \mathrm{~km}$ até a embocadura no Oceano Atlântico, no município de São João da Barra (Fig. 1).

Em cada local utilizaram-se as seguintes artes de pesca: tarrafa, com diâmetro de $3 \mathrm{~m}$ e malha $2 \mathrm{~cm}$ entre nós; redes de espera, com malhas de 2,5 a $6,5 \mathrm{~cm}$ entre nós opostos, comprimento de $30 \mathrm{~m}$ e altura de $2 \mathrm{~m}$; e peneira com diâmetro de $80 \mathrm{~cm}$ e malha de $1 \mathrm{~mm}$. Um total de 22 redes foi colocado em cada local de amostragem, com permanência desde a tarde de um dia até a manhã do dia seguinte. A unidade amostral, utilizada para comparações entre os locais e os períodos foi estabelecida como o total de peixes capturado nas 22 redes de espera, acrescidos de 5 séries de 20 lances de tarrafas, e 5 séries de 20 lances de peneiras, com estes equipamentos de pesca tendo sido utilizados aleatoriamente ao longo de um trecho de aproximadamente $800 \mathrm{~m}$ de extensão, durante as 24 horas de permanência no local amostrado.

Os peixes coletados foram fixados em formalina $10 \%$ por 48 horas e, posteriormente, transferidos para álcool 70\%. Após triagem e identificação, o material foi depositado na coleção de referência do Laboratório de Ecologia de Peixes da UFRRJ. A identificação dos peixes teve por base a compilação de REIS et al. (2003), além de uma literatura variada mais específica para peixes neotropicais, tendo incluído também a consulta a especialistas.

Os peixes foram pesados (em $0,1 \mathrm{~g}$ ) e medidos no comprimento total $(\mathrm{em} 0,1 \mathrm{~cm})$ individualmente. Foram determinados os seguintes índices: riqueza de espécies de Margalef, diversidade de Shannon-Wiener, dominância de Simpson (SI) e equitabilidade (PIELOU, 1966). Todos estes índices encontram-se descritos em MAGURRAN (1988), e foram calculados considerando o logaritmo na base decimal.

Comparações das médias do número de indivíduos e de espécies por unidade amostral, bem como dos índices de diversidade e da abundância das espécies dominantes (>1\% do total capturado) foram feitas entre os quatro trechos do rio (unidades geográficas) e entre os períodos de cheia e de seca, utilizando o teste não-paramétrico de Kruskal-Wallis. Quando diferenças significativas foram encontradas, foi utilizado o teste de Mann-Whitney ( $\mathrm{P}<$ 0,05) para comparações caso-a-caso. Estes testes nãoparamétricos foram utilizados em substituição à ANOVA (Análise de Variância), uma vez que os dados não atenderam os requisitos de homocedasticidade e normalidade (ZAR, 1999).

\section{RESULTADOS}

Foram coletados 8.570 indivíduos, pertencentes a 9 ordens, 29 famílias, 55 gêneros e 81 espécies (Tab. II). Characiformes predominaram com 28 (34,6\%) espécies, seguidos por Siluriformes com 23 (28,4\%). Perciformes foram representados por 19 espécies, sendo 8 marinhas, enquanto os grupos menos representados foram Clupeiformes, Elopiformes, Gymnotiformes, Cyprinodontiformes, Synbranchiformes e Pleuronectiformes. Characidae foi a família de maior diversidade com 15 espécies, seguida de Loricariidae com 11 espécies.

Vinte espécies (Tab. III) apresentaram contribuição numérica acima de $1 \%$ e contribuíram com $87,4 \%$ da captura do número de indivíduos e $74,7 \%$ do peso total, tendo sido utilizadas para comparações espaço-temporais. As cinco espécies mais abundantes contribuíram com $45,8 \%$ do número total de indivíduos e $16,9 \%$ do peso total. Vinte e cinco espécies apresentaram número de indivíduos inferior a $0,1 \%$ do total capturado e 26 apresentaram peso inferior a $0,1 \%$ do total de peixes (Tab. III). As espécies de maior participação em peso foram H. luetkeni, G. brasiliensis, $P$. maculatus, $P$. lineatus e $H$. littorale. Somente 3 espécies ( $G$. brasiliensis, $O$. hepsetus e A. bimaculatus) foram classificadas como constantes nas amostragens, isto é, ocorreram em mais de $75 \%$ das amostras.

Cichlidae foi a família mais abundante com $28 \%$ do número total de indivíduos, contribuindo com $8 \%$ em número de espécies e $16 \%$ do peso total capturado (Tab. III). Characidae contribuiu com 19\% do número de indivíduos, e com $12 \%$ do peso total. As ordens que apresentaram maior contribuição numérica para o total de peixes capturados foram Characiformes $(29,5 \%)$, Perciformes (28\%), Siluriformes (24\%) e Cyprinodontiformes $(17 \%)$; estes últimos são constituídos por peixes de pequeno porte, variando de 9 a $36 \mathrm{~mm}$ de comprimento total. Elopiformes, Clupeiformes, Gymnotiformes, Synbranchiformes e Pleuronectiformes apresentaram menor contribuição, todas com menos de $1 \%$ do número de peixes coletados.

Um maior número de espécies em todas as ordens foi observado durante o período de cheias. Também observou-se um aumento da riqueza ao longo do trecho longitudinal do rio, com menores valores no trecho superior e maiores no trecho inferior. Characiformes e Siluriformes foram os grupos predominantes nos trechos superior e médio-superior. Os trechos médioinferior e inferior apresentaram predominância de Characiformes, Siluriformes e Perciformes, em proporções aproximadamente iguais (Tab. IV).

As espécies abundantes amplamente distribuídas nos quatro trechos da bacia foram $O$. hepsetus, $G$. brasiliensis e $P$. reticulata; também apresentaram ampla distribuição espécies residentes, como $H$. affinis, $R$. quelen e A. giton (Tab. V), que não diferiram significativamente na abundância entre os quatro trechos do rio. Por outro lado, apresentaram diferenças significativas entre os trechos $H$. littorale, que foi significativamente mais abundante no trecho médiosuperior; P. fur, H. luetkeni, P. vivipara, G. albescens, $C$. gilberti, no trecho médio-inferior e $L$. spixii e $P$. lineatus, no trecho inferior (Tab. V; Fig. 2).

O número total de indivíduos foi maior durante o período chuvoso, correspondendo a $64,8 \%$ do total de peixes coletados. Poecilia vivipara foi a única espécie abundante a ocorrer somente no período de seca, tendo se distribuído em todos os trechos, com exceção do superior. O trecho médio-superior apresentou número de indivíduos mais elevado durante o período de cheia, embora com erro padrão relativamente elevado devido à variabilidade numérica entre as amostras; as elevadas 
Tabela II. Lista de espécies de peixes agrupadas por ordem e família, coletadas no rio Paraíba do Sul, entre os anos de 2002 e 2003 (*, espécies não nativas).

\begin{tabular}{|c|c|c|}
\hline Ordem & Família & Espécie \\
\hline Elopiformes & Elopidae & Elops saurus Linnaeus, 1766 \\
\hline \multirow{2}{*}{ Clupeiformes } & Engraulidae & Anchoviella lepidentostole (Fowler, 1911) \\
\hline & & Lycengraulis grossidens (Agassiz, 1829) \\
\hline \multirow[t]{28}{*}{ Characiformes } & Anostomidae & Abramites hypselenotus (Gunther, 1868)* \\
\hline & & Leporinus conirostris (Steindachner, 1875) \\
\hline & & Leporinus copelandii (Steindachner, 1875) \\
\hline & & Leporinus mormyrops (Steindachner, 1875) \\
\hline & & Leporinus sp. \\
\hline & Serrasalmidae & Metynnis maculatus (Kner, 1858)* \\
\hline & Characidae & Astyanax bimaculatus (Linnaeus, 1758) \\
\hline & & Astyanax giton (Eigenmann, 1908) \\
\hline & & Astyanax parahybae Eigenmann, 1908 \\
\hline & & Astyanax scabripinnis (Jenyns, 1842) \\
\hline & & Astyanax sp.1 \\
\hline & & Astyanax sp.2 \\
\hline & & Deuterodon sp.1 \\
\hline & & Deuterodon $\mathrm{sp} .2$ \\
\hline & & Brycon insignis Steindachner, 1877 \\
\hline & & Brycon sp. \\
\hline & & Hyphessobrycon bifasciatus (Ellis, 1991) \\
\hline & & Hyphessobrycon callistus (Boulenger, 1900)* \\
\hline & & Oligosarcus hepsetus (Cuvier, 1829) \\
\hline & & Probolodus heterostomus Eigenmann, 1911 \\
\hline & & Salminus brasiliensis (Cuvier, 1816)* \\
\hline & Crenuchidae & Characidium sp. 1 \\
\hline & & Characidium sp. 2 \\
\hline & Erythrinidae & Hoplias malabaricus (Bloch, 1794) \\
\hline & & Hoplyeritrinus unitaeniatus (Agassiz, 1829) \\
\hline & Prochilodontidae & Prochilodus lineatus (Valenciennes, 1836) \\
\hline & & Prochilodus vimboides Kner, 1859 \\
\hline & Curimatidae & Cyphocharax gilbert (Quoy \& Gaimard, 1824) \\
\hline \multirow[t]{23}{*}{ Siluriformes } & Auchenipteridae & Glanidium albescens Reinhardt, 1824 \\
\hline & & Trachelyopterus striatulus (Steindachner, 1877) \\
\hline & Ariidae & Genidens genidens (Valenciennes, 1839) \\
\hline & Pimelodidae & Pimelodus maculatus (Lacépède, 1803) \\
\hline & & Pimelodus fur (Reinhardt, 1874) \\
\hline & Heptapteridae & Pimelodella eigenmanni (Boulenger, 1891) \\
\hline & & Rhamdia quelen (Quoy \& Gaimard, 1824) \\
\hline & & Rhamdia sp.1 \\
\hline & Callichthyidae & Callichthys callichthys (Linnaeus, 1758) \\
\hline & & Corydoras nattereri (Steindachner, 1876) \\
\hline & & Hoplosternum littorale (Hancock, 1828) \\
\hline & Clariidae & Clarias gariepinus (Burchell, 1822)* \\
\hline & Loricariidae & Hemipsilichthys gobio (Lutken, 1874) \\
\hline & & Hemipsilichthys sp. \\
\hline & & Hypostomus affinis (Steindachner, 1876) \\
\hline & & Hypostomus luetkeni (Steindachner, 1876) \\
\hline & & Hypostomus sp.1 \\
\hline & & Loricariichthys spixii (Steindachner, 1877) \\
\hline & & Harttia loricariformes (Steindachner, 1876) \\
\hline & & Pogonopoma parahybae (Steindachner, 1877) \\
\hline & & Rineloricaria cf. lima (Kner, 1953) \\
\hline & & Rineloricaria steindachneri (Regan, 1904) \\
\hline & & Rineloricaria sp.1 \\
\hline \multirow[t]{2}{*}{ Gymnotiformes } & Gymnotidae & Gymnotus cf. carapo Linnaeus, 1758 \\
\hline & Sternopygidae & Eigenmannia virescens (Valenciennes, 1847) \\
\hline \multirow[t]{3}{*}{ Cyprinodontiformes } & Poecilidae & Phalloceros caudimaculatus (Hensel, 1868) \\
\hline & & Poecilia reticulata (Peters, 1859)* \\
\hline & & Poecilia vivipara Bloch \&Schneider, 1801* \\
\hline Synbranchiformes & Synbranchidae & Synbranchus marmoratus Bloch, 1795 \\
\hline \multirow[t]{19}{*}{ Perciformes } & Cichlidae & Aequidens sp.* \\
\hline & & Cichla monoculus Spix \& Agassiz, 1831* \\
\hline & & Cichla ocellaris Bloch \& Schneider, $1801^{*}$ \\
\hline & & Crenicichla lacustris (Castelnau, 1855) \\
\hline & & Cichlasoma cf. facetum Jenys, 1842 \\
\hline & & Cichlasoma sp. \\
\hline & & Geophagus brasiliensis (Quoy \& Gaimard, 1824) \\
\hline & & Tilapia rendalli (Boulenger, 1896)* \\
\hline & & Oreochromis niloticus (Linnaeus, 1758$)^{*}$ \\
\hline & Centropomidae & Centropomus parallelus (Poey, 1860) \\
\hline & & Centropomus undecimalis (Bloch, 1792) \\
\hline & Mugilidae & Mugil curema (Valenciennes, 1836) \\
\hline & & Mugil liza (Valenciennes, 1836) \\
\hline & Polycentridae & Polycentrus schomburgkii Muller \& Troschel, 1849 \\
\hline & Scianidae & Pachyurus adspersus Steindachner, 1879 \\
\hline & & Plagioscion squamosissimus (Heckel, 1840)* \\
\hline & Carangidae & Caranx latus (Agassiz, 1831) \\
\hline & & Caranx crysos (Mitchill, 1815) \\
\hline & Gobidae & Awaous tajasica (Lichtenstein, 1822) \\
\hline \multirow{2}{*}{ Pleuronectiformes } & Achiridae & Trinectes paulistanus (Miranda-Ribeiro, 1915) \\
\hline & Paralichthyidae & Citharichthys spilopterus Günther, 1862 \\
\hline
\end{tabular}


Tabela III. Número total (N), peso (em gramas), freqüências de ocorrência (FO) e amplitude de tamanho de peixes (ACt, mm) no rio Paraíba do Sul, $2002 / 2003$.

\begin{tabular}{|c|c|c|c|c|c|c|}
\hline Espécie & Número & $\mathrm{N} \%$ & Peso & $\mathrm{P} \%$ & $\% \mathrm{FO}$ & $\mathrm{ACt}$ \\
\hline T. rendalli & 1290 & 15 & 4641 & 1,1 & 33,3 & $12-390$ \\
\hline G. brasiliensis & 780 & 9,1 & 39030 & 9,2 & 85,7 & $10-430$ \\
\hline P. reticulata & 547 & 6,4 & 104 & $<0,1$ & 23,8 & $11-35$ \\
\hline P. vivipara & 528 & 6,1 & 118 & $<0,1$ & 21,4 & $10-36$ \\
\hline O. hepsetus & 527 & 6,1 & 27889 & 6,6 & 90,5 & $26-285$ \\
\hline P. lineatus & 496 & 5,8 & 29870 & 7 & 33,3 & $180-255$ \\
\hline A . bimaculatus & 447 & 5,2 & 8772 & 2 & 78,6 & $110-175$ \\
\hline P. maculatus & 403 & 4,7 & 31598 & 7,5 & 50,0 & $160-316$ \\
\hline P. caudimaculatus & 378 & 4,4 & 149 & $<0,1$ & 35,7 & $9-35$ \\
\hline A. parahybae & 317 & 3,7 & 8295 & 2 & 64,3 & $45-160$ \\
\hline H. littorale & 272 & 3,2 & 29653 & 7 & 59,5 & $115-243$ \\
\hline P. fur & 265 & 3,1 & 14015 & 3,3 & 33,3 & $150-250$ \\
\hline H. luetkenii & 253 & 2,9 & 41960 & 9,9 & 57,1 & $100-400$ \\
\hline G. albescens & 245 & 2,9 & 11247 & 2,7 & 40,5 & $111-151$ \\
\hline C. gilbert & 166 & 1,9 & 10853 & 2,6 & 35,7 & $110-188$ \\
\hline H. affinis & 153 & 1,8 & 27399 & 6,5 & 73,8 & $30-405$ \\
\hline A. giton & 129 & 1,5 & 2494 & 0,6 & 26,2 & $98-140$ \\
\hline L. spixii & 121 & 1,4 & 12939 & 3,1 & 21,4 & $250-450$ \\
\hline C. lacustris & 100 & 1,2 & 4768 & 1,1 & 45,2 & $145-280$ \\
\hline R. quelen & 95 & 1,1 & 11359 & 2,7 & 33,3 & $20-700$ \\
\hline E. virescens & 73 & 0,9 & 2730 & 0,6 & 50,0 & $210-367$ \\
\hline L. copelandii & 70 & 0,8 & 23102 & 5,5 & 42,9 & $260-450$ \\
\hline L. mormyrops & 66 & 0,8 & 3803 & 0,9 & 21,4 & $170-215$ \\
\hline H. malabaricus & 60 & 0,7 & 20785 & 4,9 & 57,1 & $50-335$ \\
\hline H. callisthus & 57 & 0,7 & 12 & $<0,1$ & 21,4 & 28-39 \\
\hline T. striatulus & 53 & 0,6 & 3835 & 0,9 & 38,1 & $150-190$ \\
\hline Rineloricaria sp.1 & 49 & 0,6 & 663 & 0,1 & 31,0 & $40-80$ \\
\hline Aequidens sp. & 46 & 0,5 & 471 & 0,1 & 11,9 & $100-150$ \\
\hline C. nattereri & 43 & 0,5 & 177 & $<0,1$ & 9,5 & $25-86$ \\
\hline Cichlasoma sp. & 39 & 0,5 & 443 & 0,1 & 9,5 & $20-70$ \\
\hline G. carapo & 36 & 0,4 & 2452 & 0,6 & 50,0 & $196-300$ \\
\hline O. nilotica & 34 & 0,4 & 14658 & 3,5 & 23,8 & $240-321$ \\
\hline A. scabripinnis & 32 & 0,4 & 490 & 0,1 & 7,1 & $111-130$ \\
\hline Deuterodo sp.1 & 32 & 0,4 & 413 & 0,1 & 16,7 & $100-130$ \\
\hline H. loricariforme & 30 & 0,3 & 871 & 0,2 & 16,7 & $150-160$ \\
\hline P. adspersus & 27 & 0,3 & 2092 & 0,5 & 16,7 & $160-205$ \\
\hline Astyanax sp. 1 & 26 & 0,3 & 680 & 0,2 & 9,5 & $100-120$ \\
\hline H. bifasciatus & 25 & 0,3 & 7 & $<0,1$ & 4,8 & $20-40$ \\
\hline C.cf.. facetum & 23 & 0,3 & 932 & 0,2 & 26,2 & $85-160$ \\
\hline A. tajasica & 23 & 0,3 & 366 & 0,1 & 9,5 & $30-145$ \\
\hline C. paralellus & 17 & 0,2 & 6518 & 1,5 & 16,7 & $290-500$ \\
\hline C. callichthys & 16 & 0,2 & 702 & 0,2 & 7,1 & $110-230$ \\
\hline P. heterostomus & 16 & 0,2 & 442 & 0,1 & 14,3 & $100-122$ \\
\hline H. gobio & 16 & 0,2 & 652 & 0,2 & 4,8 & $115-180$ \\
\hline Astyanax sp. 2 & 13 & 0,2 & 164 & $<0,1$ & 2,4 & $100-110$ \\
\hline Rhamdia sp.1 & 16 & 0,1 & 856 & 0,2 & 9,5 & $20-30$ \\
\hline A. hypselenotus & 12 & 0,1 & 15 & $<0,1$ & 7,1 & $10-12$ \\
\hline R. cf. . lima & 10 & 0,1 & 98 & $<0,1$ & 9,5 & $150-190$ \\
\hline Hypostomus sp.1 & 10 & 0,1 & 599 & 0,1 & 4,8 & $50-60$ \\
\hline H. unitaeniatus & 9 & 0,1 & 765 & 0,2 & 9,5 & $221-260$ \\
\hline L. grossidens & 7 & 0,1 & 621 & 0,1 & 4,8 & $220-250$ \\
\hline M. maculatus & 7 & 0,1 & 259 & 0,1 & 7,1 & $124-135$ \\
\hline P. vimboides & 7 & 0,1 & 1169 & 0,3 & 7,1 & $150-195$ \\
\hline Leporinus sp. & 7 & 0,1 & 500 & 0,1 & 2,4 & $150-200$ \\
\hline L. conirostris & 6 & 0,1 & 1775 & 0,4 & 11,9 & $315-350$ \\
\hline C. monoculos & 5 & 0,1 & 1773 & 0,4 & 11,9 & $270-320$ \\
\hline M. curema & 3 & $<0,1$ & 299 & 0,1 & 7,1 & $214-580$ \\
\hline M. liza & 3 & $<0,1$ & 2777 & 0,7 & 2,4 & $270-317$ \\
\hline S. brasiliensis & 3 & $<0,1$ & 1187 & 0,3 & 4,8 & $336-400$ \\
\hline G. genidens & 3 & $<0,1$ & 496 & 0,1 & 4,8 & $280-322$ \\
\hline T. paulistanus & 3 & $<0,1$ & 39 & $<0,1$ & 2,4 & $84-91$ \\
\hline Brycon sp. & 2 & $<0,1$ & 455 & 0,1 & 2,4 & $150-173$ \\
\hline C. ocelaris & 2 & $<0,1$ & 187 & $<0,1$ & 4,8 & $135-145$ \\
\hline Characidium sp. 2 & 2 & $<0,1$ & $<0,1$ & $<0,1$ & 2,4 & $20-30$ \\
\hline P. parahybae & 2 & $<0,1$ & 1047 & 0,2 & 4,8 & $210-232$ \\
\hline R. steindachneri & 2 & $<0,1$ & 1 & $<0,1$ & 2,4 & $40-45$ \\
\hline A. lepidentostole & 1 & $<0,1$ & 31 & $<0,1$ & 2,4 & 145 \\
\hline B. insignis & 1 & $<0,1$ & 52 & $<0,1$ & 2,4 & 185 \\
\hline C. latus & 1 & $<0,1$ & 24 & $<0,1$ & 2,4 & 112 \\
\hline Characidium sp. 1 & 1 & $<0,1$ & 2 & $<0,1$ & 2,4 & 64 \\
\hline C. undecimalis & 1 & $<0,1$ & 1950 & $<0,1$ & 2,4 & 485 \\
\hline Deuterodon sp.1 & 1 & $<0,1$ & 17 & $<0,1$ & 2,4 & 112 \\
\hline E. saurus & 1 & $<0,1$ & 135 & $<0,1$ & 2,4 & 300 \\
\hline P. eigenmanni & 1 & $<0,1$ & 20 & $<0,1$ & 2,4 & 135 \\
\hline P. squamosissimus & 1 & $<0,1$ & 164 & $<0,1$ & 2,4 & 230 \\
\hline P. schombrugkii & 1 & $<0,1$ & $<0,1$ & $<0,1$ & 2,4 & 27 \\
\hline C. crysos & 1 & $<0,1$ & 145 & $<0,1$ & 2,4 & 220 \\
\hline S. marmoratus & 1 & $<0,1$ & 25 & $<0,1$ & 2,4 & 152 \\
\hline C. spilopterus & 1 & $<0,1$ & 14 & $<0,1$ & 2,4 & 125 \\
\hline Hemipsilichthys sp & 1 & $<0,1$ & 34 & $<0,1$ & 2,4 & 135 \\
\hline C. gariepinnus & 1 & $<0,1$ & 1018 & 0,2 & 2,4 & 495 \\
\hline
\end{tabular}

Iheringia, Sér. Zool., Porto Alegre, 95(4):347-357, 30 de dezembro de 2005 
Tabela IV. Número de espécies de peixes por unidade geográfica durante os períodos de cheia (C) e seca (S), no rio Paraíba do Sul, 2002/ 2003.

\begin{tabular}{|c|c|c|c|c|c|c|c|c|c|}
\hline & \multicolumn{2}{|c|}{ Superior } & \multicolumn{2}{|c|}{ Médio-Superior } & \multicolumn{2}{|c|}{ Médio-Inferior } & \multicolumn{2}{|c|}{ Inferior } & Total \\
\hline & $\mathrm{C}$ & $\mathrm{S}$ & $\mathrm{C}$ & $\mathrm{S}$ & $\mathrm{C}$ & $\mathrm{S}$ & $\mathrm{C}$ & $\mathrm{S}$ & \\
\hline Characiformes & 14 & 11 & 9 & 8 & 14 & 10 & 17 & 11 & 28 \\
\hline Siluriformes & 7 & 9 & 12 & 7 & 12 & 11 & 13 & 9 & 23 \\
\hline Perciformes & 4 & 3 & 4 & 3 & 15 & 9 & 12 & 6 & 19 \\
\hline Cyprinodontiformes & 2 & 1 & 2 & 3 & 1 & 2 & 2 & 2 & 3 \\
\hline Outros & 0 & 0 & 2 & 1 & 4 & 4 & 2 & 2 & 8 \\
\hline Total & 27 & 24 & 29 & 22 & 46 & 36 & 46 & 30 & 81 \\
\hline
\end{tabular}

abundâncias de $T$. rendalli neste trecho foram responsáveis por este padrão.

O número de indivíduos e o número de espécies por amostra não apresentaram diferenças significativas $(\mathrm{P}>0,05)$ entre os períodos seca/cheia nem entre os trechos do rio, embora algumas tendências tenham sido observadas (Fig. 3). Na seca verificou-se uma tendência de aumento da abundância de peixes do trecho superior para o trecho inferior; o número de espécies por amostra seguiu esta mesma tendência, sendo mais evidente no período de cheia.

Os quatro índices de diversidade apresentaram diferentes tendências ao longo das diferentes unidades geográficas. A riqueza de Margalef seguiu o mesmo padrão do número de espécies/amostra, crescendo do

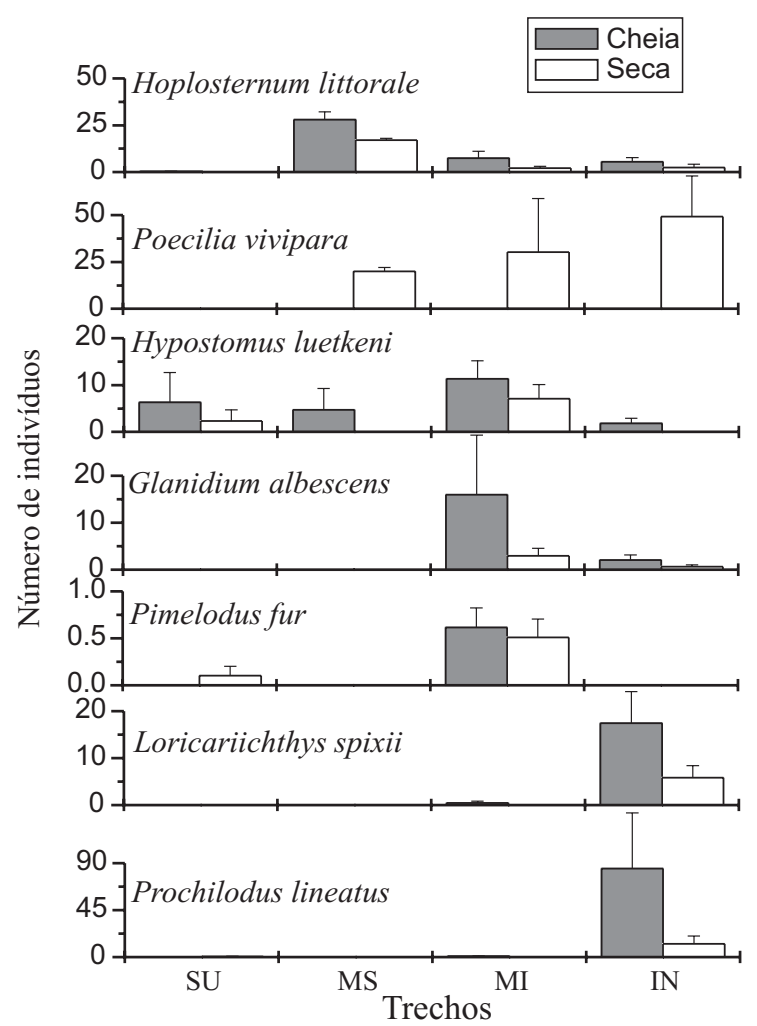

Fig. 2. Médias e erros padrões (linhas verticais) da abundância das espécies que apresentaram diferenças significativas entre os trechos (SU, superior; MS, médio superior; MI, médio inferior; IN, inferior) e por período do ano (cheia/seca) no rio Paraíba do Sul, 2002/ 2003. trecho superior para o trecho inferior, principalmente no período de cheia. No período de seca, apenas o trecho superior apresentou menores valores.

O índice de diversidade de Shannon-Wiener foi mais elevado nos trechos superior e médio-superior no período de seca, ocorrendo o oposto no período chuvoso. A dominância de Simpson no trecho superior e médiosuperior foi menor na seca, com o contrário ocorrendo no trecho médio-inferior e inferior.

A equitabilidade no período de seca foi mais elevada no trecho superior e médio-superior, enquanto no período chuvoso também observou-se maior média no trecho superior, diminuindo acentuadamente no trecho médiosuperior e mantendo-se em níveis intermediários nos trechos médio-inferior e inferior.
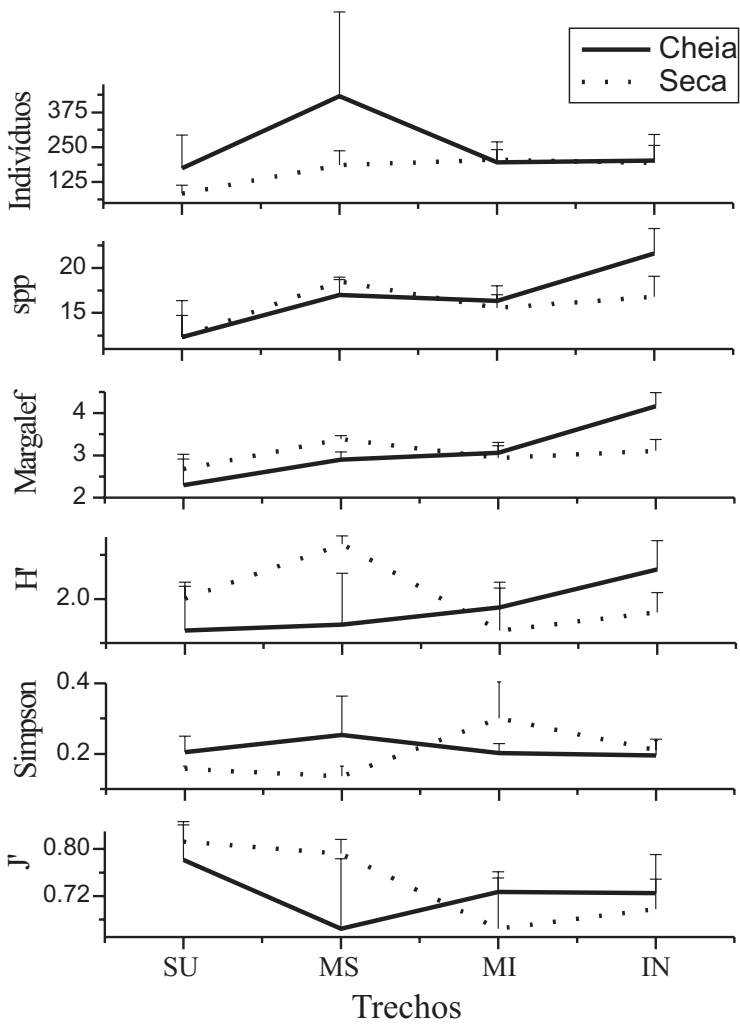

Fig. 3. Médias e erros padrões (linhas verticais) do número de indivíduos, número de espécies, riqueza de Margalef, Índice de diversidade de Shannon-Wiener (H'), Simpson e Equitabilidade (J') por trechos (SU, superior; MS, médio superior; MI, médio inferior; IN, inferior) e por período do ano (cheia/seca) no rio Paraíba do Sul, 2002/2003. 
Tabela V. Comparação do número das 20 espécies mais abundantes no rio Paraíba do Sul (2002/2003), entre os trechos (SU, superior; MS, médio superior; MI, médio inferior; IN, inferior) e períodos do ano (cheia/seca), através do teste não-paramétrico de KruskallWallis $(\mathrm{H})$, e teste a posteriori de Mann-Whitney (U). n.s., não significativo; *, significativo $(\mathrm{P}<0,05)$; **, altamente significativo $(\mathrm{P}<0,01)$.

\begin{tabular}{|c|c|c|}
\hline Espécies & $\mathrm{H}$ & $\mathrm{U}$ \\
\hline T. rendalli & n.s. & - \\
\hline G. brasiliensis & n.s. & - \\
\hline P. reticulata & n.s. & - \\
\hline O. hepsetus & n.s. & - \\
\hline A. bimaculatus & n.s. & - \\
\hline P. fur & $15,96 * *$ & $\mathrm{MI}>\mathrm{SU} ; \mathrm{MS} ; \mathrm{IN}$ \\
\hline C. lacustris & $9,01 *$ & - \\
\hline P. caudimaculatus & $11,05 *$ & - \\
\hline A. paraybae & n.s. & - \\
\hline P. lineatus & $9,51 *$ & IN > SU; MS; MI \\
\hline P. vivipara & $9,97 *$ & - \\
\hline H. littorale & $13,02 * *$ & $\mathrm{MS}>\mathrm{MI} ; \mathrm{IN}>\mathrm{SU}$ \\
\hline G. albescens & $10,27 *$ & MI; IN > SU; MS \\
\hline H. luetkeni & $11,82 * *$ & MI; SU > MS; IN \\
\hline C. gilberti & $9,89 *$ & - \\
\hline H. affinis & n.s. & - \\
\hline A. giton & n.s. & - \\
\hline L. spixii & $26,83 * *$ & IN > SU; MS; MI \\
\hline R. quelen & n.s. & - \\
\hline
\end{tabular}

\section{DISCUSSÃO}

O rio Paraíba do Sul, apesar de toda sua história de alterações ao longo de mais de quatro séculos de exploração e de sua localização entre os maiores centros urbano-industriais do país, ainda é depositário de uma biodiversidade relativamente elevada. Em geral, a comunidade de peixes em grandes rios apresenta elevada diversidade, que reflete a diversidade estrutural e riqueza de habitats das zonas litorâneas e a conectividade com áreas de inundações (Schiemer, 2000), o que foi confirmado para o caso do rio Paraíba do Sul. As espécies registradas neste trabalho indicam a grande importância de conservação deste sistema para a ecologia da região.

Relações de espécies têm sido reportadas para o rio Paraíba do Sul como parte de compilações, porém sem precisar os locais e períodos de amostragens (BIzERRIL, 1999); outras são restritas a determinados trechos (ARAúJo, 1996; ARAúJo et al., 2001), não fornecendo um quadro abrangente do sistema. Por outro lado, as 92 espécies de água doce para este sistema, incluídas na compilação de Fowler $(1948,1950,1951,1954)$, fornecem um quadro mais compreensivo da riqueza do rio. A estimativa de uma riqueza de 130 espécies para grandes rios da bacia do Leste, como o Paraíba do Sul, foi feita por BizerRil (1999), incluindo o levantamento da ictiofauna de pequenos riachos, dos quais, vários componentes não chegam a ocorrer no canal principal. ARAúJo (1996) registrou 57 espécies de água doce entre os trechos médio-inferior e inferior; ARAúJo et al. (2001) registraram 52 espécies para o canal principal do rio entre os municípios de Barra Mansa e Barra do Piraí, um trecho considerado de maior nível de poluição por PFEIFFER et al. (1986). Comparações da riqueza de espécies entre trabalhos com diferentes níveis de esforço podem ser pouco confiáveis do ponto de vista quantitativo, embora tenham grande importância como informação qualitativa.
Ecoregiões têm sido definidas como unidades geográficas que geralmente exibem similaridades no mosaico dos recursos ambientais, ecossistemas e efeitos do homem (BAILEY, 1983). Embora este conceito ainda não tenha sido aplicado no Brasil, uma separação das condições ambientais parece ocorrer ao longo do rio Paraíba do Sul. O trecho superior, com altitude entre 500 e 1800 metros, onde se encontram as nascentes do rio, apresenta clima tropical de altitude, declividade elevada e variável, determinando o caráter dinâmico do sistema fluvial, com presença de corredeiras, características de regiões de serras e planaltos. Tais condicionantes ambientais diferem do trecho relativamente plano que compreende o médio-superior e médio-inferior. Estes dois trechos apresentam maior diversidade na fisiografia, com o rio apresentando desde meandros e áreas planas inundáveis onde podem ser observadas lagoas marginais, no trecho médio-superior, até extensões mais encaixadas do trecho médio-inferior já nas proximidades do trecho inferior; o clima é mesotérmico, com verões quentes e chuvosos e invernos secos. $\mathrm{O}$ trecho inferior caracterizase por um número elevado de sistemas lênticos (brejos, lagos e lagoas) que, em muitos casos, são alimentados pelo transbordamento do rio Paraíba do Sul, com pequena declividade e temperaturas elevadas; observa-se um expressivo alargamento da planície aluvial, notadamente nas proximidades do delta na planície dos Goytacazes. Portanto, apesar de não ocorrer uma definida separação das unidades geográficas devido à ampla diferenciação de paisagens e dos hábitats ao longo da bacia, é possível que algumas espécies apresentem hábitats preferenciais e restritos.

As diferenciações ambientais ao longo do percurso longitudinal, representadas pelas quatro unidades geográficas e pelas barreiras físicas (barramentos) não parecem limitar a distribuição das espécies, não somente das mais abundantes, como também das residentes, que são encontradas ao longo de quase toda a extensão deste sistema. Quatro grandes reservatórios foram construídos para produção de energia hidrelétrica neste sistema, dois no trecho superior (Paraibuna e Santa Branca), um próximo do limite entre o trecho médio-superior e médioinferior (Funil), e o outro nas proximidades do limite entre o trecho médio-inferior e o trecho inferior (Ilha dos Pombos). Tais reservatórios, embora contribuam para diminuição da riqueza de espécies do sistema devido à fragmentação do habitat, não impediram que a maioria das espécies abundantes tenha apresentado ampla distribuição espacial. Estas espécies são de grande plasticidade trófica, sendo pequenos forrageiros, principalmente insetívoros, com tendência a omnivoria. Espécies como T. rendalli, G. brasiliensis, $P$. vivipara, $P$. reticulata e $O$. hepsetus têm a característica comum de não apresentarem especialização trófica muito definida, consumindo ítens de maior oferta no ambiente. Exceção pode ser feita para $O$. hepsetus que possui hábitos alimentares predominantemente carnívoros, consumindo insetos, larvas e pequenos peixes (HARTZ et al., 1986). Embora esta última autora tenha estudado uma outra espécie do gênero Oligosarcus, não são esperadas diferenças nos hábitos alimentares de espécies congenéricas (HeLfman et al., 1997). Segundo KNOPPEL 
(1970), a grande plasticidade trófica de espécies oportunistas pode ser vista como uma expressão da capacidade de utilizar uma diversidade de recursos alimentares em condições adversas, onde espécies menos tolerantes não seriam capazes de utilizar.

Poecilidae registrados no presente trabalho são peixes amplamente conhecidos por sua capacidade de tolerar ambientes alterados, ocorrendo em locais onde um grande número de espécies tem distribuição limitada (ArAújo et al., 2003). No Paraíba do Sul, são um dos grupos mais abundantes e amplamente distribuídos. ARANHA \& CARAMASCHI (1999) verificaram que este grupo de peixes apresenta período reprodutivo prolongado e atribuem esta estratégia a uma resposta biológica a ambientes instáveis, onde a reposição contínua de jovens constituiria um mecanismo de restabelecimento da estrutura da população.

A diversidade de hábitats influencia a disponibilidade dos recursos com a ocupação e a permanência das espécies nos biótopos, sendo dependente da habilidade e exigência de cada espécie (MARTIN-SMith, 1998). A capacidade de distribuição das espécies ao longo do trecho longitudinal do rio pode estar associada com a habilidade de realizar migrações ou movimentos mais amplos. No presente estudo, Siluriformes predominaram entre as espécies que tiveram distribuição restrita a determinados trechos, com $H$. littorale tendo sido associado ao trecho médio superior, $P$. fur, G. albescens e $H$. luetkeni ao médio inferior e $L$. spixii ao trecho inferior. Estes peixes possuem corpo achatado, são proximamente associados ao fundo e possuem pequena capacidade de realização de movimentos longitudinais (AXELROD \& KULLANDER, 2003). A ocorrência em grandes números de L. spixii no trecho inferior do rio Paraíba do Sul foi anteriormente reportada por Araúso (1996), coincidindo com o encontrado no presente trabalho. Por outro lado, P. lineatus, uma espécie eminentemente migratória, apresentou distribuição mais restrita ao trecho inferior, ocorrendo ocasionalmente em outros trechos. Esta espécie pertence a um gênero de peixes que realiza migrações reprodutivas rio acima (piracema) em vários sistemas lóticos brasileiros (Godoy, 1962) e que tem nas zonas baixas do Paraíba do Sul suas áreas de alimentação.

O trecho superior foi o único onde não se registrou predomínio de nenhuma espécie em particular, quando comparado com os outros trechos, sendo também, como esperado, o que apresentou menor riqueza e abundância de espécies. Correntes de cabeceira normalmente apresentam pequeno volume de água, são rasas e possuem cursos irregulares (WhitTon, 1975). Durante períodos de chuvas, tais áreas tornam-se difíceis de serem amostradas, o que pode estar associado às menores riquezas e abundâncias encontradas.

$\mathrm{O}$ trecho inferior apresentou o maior número de espécies, contrastando com o trecho superior, e corroborando a teoria do rio contínuo, onde é esperada maior diversidade nas zonas baixas do rio, devido, principalmente, à maior complexidade de hábitat (PIANKA, 1974; VANOTE et al., 1980). No caso particular do rio Paraíba a maior riqueza neste trecho também pode ser atribuída à proximidade da região estuarina de onde várias espécies marinhas eurihalinas ou estuarinas como $C$. parallelus, A. tajasica e L. grossidens foram registradas. As diferenciações na fisiografia ao longo dos quatro trechos do rio parecem, portanto, ter correspondido às mudanças nas assembléias de peixes, uma indicação da influência dos microhábitats na estruturação da comunidade de peixes.

Durante este trabalho (março de 2003), foi verificado e amplamente divulgado pela imprensa o acidente causado pelo rompimento da barragem de rejeitos tóxicos. Cerca de 1,2 bilhão de litros de resíduos tóxicos vazaram do reservatório da fábrica Cataguazes Indústria de Papel, no município mineiro de Cataguazes. Seus efeitos se estenderam do rio Pomba ao estuário do Paraíba do Sul e zona costeira adjacente, numa extensão de cerca de 150 quilômetros de rios, deixando mais de 600 mil pessoas sem água (Eliane Lobato, Revista Isto É, no 1749, 09/04/ 2003). Não obstante o grande impacto causado na semana do acidente, uma elevada riqueza de espécies neste trecho do rio (Campos, 27 espécies; São João da Barra, 22 espécies) foi verificado apenas duas semanas após este fato, quando comparadas com informações históricas do rio para este trecho (ARAúJo, 1996). Segundo Odum (2001) a diversidade tende a ser reduzida quando ocorre estresse ou alterações no ambiente aquático, porém no caso do rio Paraíba do Sul, a existência de grandes tributários, como o rio Muriaé, e de uma grande diversidade de hábitats, pode ter constituído áreas de refúgio para os peixes durante o período de alteração. Investigações mais detalhadas necessitariam de serem realizadas para avaliar os efeitos residuais desde acidente na ictiofauna.

A tendência de maior diversidade do trecho superior para o trecho inferior foi detectada pelo número das espécies, pela riqueza de Margalef e pela diversidade de Shannon-Wiener $\left(\mathrm{H}^{\prime}\right)$, sendo mais evidentes no período das chuvas. Por outro lado, as maiores abundâncias numéricas do trecho médio-superior, principalmente de T. rendalli, contribuiu para a diminuição da equitatividade, também no período de chuvas, embora as variações entre as amostras tenham sido muito elevadas. Neste trecho, principalmente nas proximidades do município de São José dos Campos, o rio apresenta notável grau de alteração devido à introdução de efluentes urbanos e industriais, como esgotos municipais não tratados, e à ocorrência de espécies oportunistas e não nativas, como T. rendalli, provavelmente oriundas de atividades de cultivo ou de pesca esportiva (pesque-e-pague). $\mathrm{O}$ grande número de espécimes de pequeno porte $(\mathrm{Ct}=12 \mathrm{e}$ $45 \mathrm{~mm}$ ) nesta área, principalmente tilápias, pode ser indicativo do sucesso reprodutivo desta espécie introduzida, o que pode estar causando interações negativas com outras espécies nativas.

A variação do período cheia/seca não foi um fator relevante para variação da abundância relativa da ictiofauna, embora a estação chuvosa tenha contribuído para aumentar da riqueza de espécies. O maior número de espécies encontrado no período de cheia, não está associado à diferenciação no esforço amostral de 23 locais no período de cheia e 18 locais no período de seca, com os locais adicionais do período de cheia correspondendo a trechos de difícil acesso e baixa riqueza de espécies. Os locais exclusivamente amostrados no 
período da cheia apresentaram 18 espécies, enquanto que os amostrados exclusivamente no período de seca, 20 espécies. Esta pequena diferença na riqueza confirma que a alternância/substituição dos locais de coleta entre os dois períodos não influenciou no número de espécies registradas.

Poecilia vivipara foi a única espécie que apresentou sazonalidade, sendo registrada exclusivamente no período de seca. Neste período ocorre maior exposição das margens, com eventuais diminuições da qualidade físico-química da água, devido ao menor volume e, portanto, menor capacidade de diluição de efluentes industriais ou domésticos, uma condição que poderia tornar determinados microhábitats indisponíveis para a maioria das espécies, e que $P$. vivipara poderia suportar.

As maiores abundâncias e riquezas de espécies ocorreram no período da cheia, embora diferenças significativas entre os períodos não tenham sido encontradas para a maioria das comparações. As redes de espera, por serem artes de pesca passivas, podem sofrer influência da movimentação dos peixes, mais ativa em períodos em que a temperatura é mais elevada, (Wootton, 1990). Embora o verão tenha sido o período de maiores ocorrências de chuvas, a variabilidade dos registros pluviométricos é muito grande. Segundo Barbiere \& Kronemberg (1994), o período de cheia e seca muitas vezes não apresentam diferenças significativas na pluviosidade, com o período seco apresentando normalmente maior variabilidade das chuvas. O período de cheia apresentou maior número de espécies, provavelmente devido ao aporte de matéria orgânica e sedimentos originados do lixiviamento do solo pelas chuvas, favorecendo maior oferta de alimentos. Em geral, a menor abundância dos peixes durante a seca, poderia estar associada à maior concentração dos mesmos na calha do rio, dificultando as operações de pesca. Também neste período ocorre o aumento da diversidade de hábitats, com o nível da água atingindo a vegetação marginal e colocando à disposição dos peixes abrigos e hábitats estruturalmente mais complexos, bem como recursos alimentares não disponíveis no período de seca. O conceito da descontinuidade serial estendida (JUNGWIRTH et al., 2000) associa a região de cabeceiras dos rios como mais dependente da continuidade longitudinal, os trechos médios, das interações verticais, e os trechos inferiores e planícies de inundações da conectividade lateral. A mais elevada riqueza de espécies nos trechos médio-inferior e inferior pode ser explicada pela maior diversidade de hábitats, facilitada pelos processos de conectividade lateral do rio durante os pulsos de cheias, quando comparada com o trecho superior. Também trechos altos de rios são geralmente caracterizados por serem geologicamente mais novos, formando vales estreitos, com mudanças de correntes devido ao elevado gradiente. $\mathrm{O}$ trecho baixo normalmente é muito mais velho, com um baixo gradiente, sendo caracterizado por planícies de inundação, o que facilitaria a maior riqueza de espécies, como foi o caso do rio Paraíba do Sul.

\section{CONCLUSÕES}

A biodiversidade da ictiofauna no canal principal e nos grandes tributários do rio Paraíba do Sul é representada por 81 espécies, um número comparativamente elevado em relação ao histórico deste sistema, não obstante as alterações ambientais que o rio tem passado.

As espécies abundantes amplamente distribuídas ao longo de todo o trecho longitudinal do rio (G. brasiliensis, P. reticulata e $O$. hepsetus) são oportunistas, uma possível indicação da limitação de hábitats ou da baixa qualidade ambiental do rio. Representantes dos Siluriformes foram os peixes de distribuição em áreas mais restritas, com $H$. littorale predominando no trecho médio-superior, $P$. fur, $G$. albescens e $H$. luetkeni no médio-inferior, e $L$. spixii e $P$. lineatus no inferior.

Uma tendência de aumento da riqueza de espécies no gradiente longitudinal foi constatada. Diferenciações na fisiografia ao longo da extensão longitudinal da bacia não coincidiram com mudanças nas assembléias de peixes, sugerindo que fatores associados a alterações de hábitats poderiam estar estruturando a comunidade de peixes a nível local.

Agradecimentos. Aos funcionários do Laboratório de Ecologia de Peixes da Universidade Federal Rural do Rio de Janeiro, pelo auxílio nas coletas de campo, especialmente Paulo César da Silva e Erasmo Soares dos Reis. A um revisor não identificado que, com seus comentários, muito contribuiu para a melhoria do trabalho. À Dra. Márcia Cristina Costa de Azevêdo pelas sugestões e auxílio no tratamento estatístico dos dados. Ao programa CNPq-CT-HIDRO que financiou parcialmente este projeto através do Proc. 550047-2002-6. A FAPERJ Fundação Carlos Chagas de Amparo à Pesquisa do Estado do Rio de Janeiro, por ter concedido a Bolsa Cidadão do Nosso Estado para financiamento deste projeto (Proc. E-26/152.463/ 2002).

\section{REFERÊNCIAS BIBLIOGRÁFICAS}

Amorin, M. C.; Pessoa, M. A. R. \& Medeiros, K. L. S. 1983. Otimização da rede de monitoramento no rio Paraíba do Sul. Cadernos FEEMA, Série Congressos, 16:1-36.

Aranha J. M. R. \& Caramaschi, E. P. 1999. Estrutura populacional, aspectos da reprodução e alimentação dos Cyprinodontiformes (Osteichthyes) de um riacho do sudeste do Brasil. Revista Brasileira Zoologia 16:637-651.

Araújo, F. G. 1996. Comunidade e estrutura da comunidade de peixes do médio e baixo rio Paraíba do Sul, RJ. Revista Brasileira de Biologia 56(1):111-126.

Araújo, F. G.; Fichberg, I.; Pinto, B. C. T. \& Peixoto, M. G. 2001. Variações espaciais na assembléia de peixes no rio Paraíba do Sul (Barra Mansa, Barra do Piraí), Rio de Janeiro, Brasil. Revista Brasileira de Zoologia 18(2):483-492. 2003. A preliminary index of biotic integrity for monitoring the condition of the rio Paraíba do Sul, Southeast Brazil. Environmetal Management 32(4):516-526.

Axelrod, H. R. \& Kullander. S. O. 2003. Corydoras diphyes (Siluriformes: Callichthyidae) and Otocinclus mimulus (Siluriformes: Loricariidae) two new species of catfishes from Paraguay, a case of mimetic association. Ichthyological Explorations of Freshwaters 14(3):249272

BAILEy, R. G. 1983. Delineation of ecosystems regions Environmental Management 7:365-373.

Barbiere, E. B. \& Kronemberg, D. M. P. 1994. Climatologia do litoral sul-sudeste do Estado do Rio de Janeiro. Cadernos 
de Geociências 12:57-73.

Bizerril, C. R. S. F. 1999. The ichthyofauna of the Rio Paraíba do Sul watershed. Biodiversity and biogeografic patterns. Brazilian Archives of Biology and Technology 42:233-250.

Fowler, H. W. 1948 Os peixes de água doce do Brasil (1 ${ }^{\mathrm{a}}$ entrega). Arquivos de Zoologia 6:1-204.

_. 1950. Os peixes de água doce do Brasil (2 entrega). Arquivos de Zoologia 6:205-404.

1951. Os peixes de água doce do Brasil (3ª entrega). Arquivos de Zoologia 6:405-628

1954. Os peixes de água doce do Brasil (4⿳亠丷厂 entrega). Arquivos de Zoologia, 9:1-400

Freire, A. G. \& Agostinho, A. A. 2000. Distribuição espaço temporal de oito espécies dominantes da ictiofauna da bacia do Alto Paraná. Acta Limnologica Brasiliensis 12:105-120.

Godoy, M. P. 1962. Marcação, migração e transplantação de peixes marcados na bacia do rio Paraná Superior. Arquivos do Museu Nacional 52: 105-113.

Hartz, S. M.; Martins, A. \& Barbiere, G. 1986. Dinâmica da alimentação e dieta de Oligorsacus jenynsii (Günther, 1864) na Lagoa Caconde, Rio Grande do Sul, Brasil (Teleoestei, Characidae). Boletim do Instituto de Pesca 23:21-29.

Helfman, G. S.; Collete, B. B. \& Facey, D. E. 1997. The diversity of fishes. London, Blackwell Science. 528 p.

Jungwirth, M.; Muhar, S. \& Schmutz, S. 2000. Fundamentals of fish ecological integrity and their relation to the extended serial discontinuity concept. Hydrobiologia 422/423:8597.

Knoppel, H. A. 1970. Food of central Amazonian fishes: contribution to the nutrient-ecology of Amazonian rain forest streams. Amazoniana 2:257-352.

Magurran, A. E. 1988 Ecological Diversity and Its Measurement. London, Croom Helm. 178p.

Martin-Smith, K. M. 1998. Relationships between fishes and habitat in rainforest streams in Sabah, Malaysia. Journal of Fish Biology 52: 458-482.

Meador, M. R. \& Goldstein, R. M. 2003. Assessing water quality at large geographic scales: relations among land use, water physicochemistry, riparian condition, and fish community structure. Environmental Management 31:504-517.

Odum, E. P. 2001. Fundamentos de Ecologia. Lisboa, Fundação Calouste Gulbenkian. 927p.

PiAnKa, E. R. 1974. Evolutionary Ecology. New York, Harper and Row. 356p.

Pielou, E. C. 1966. The measurement of diversity in different types of biological collections. Journal of Ecology 47:607615.

Pfeiffer, W. C.; Fiszman, M.; Malm, O. \& Azcue, J. M. 1986. Heavy metal pollution in the Paraíba do Sul River, Brasil. Science of the Total Environment 58:73-79.

Reis, R. E.; Kullander, S. O. \& Ferraris, C. J., JR. 2003. Check list of the freshwater fishes of South and Central America. Porto Alegre, Edipucrs. 742p.

Schiemer, F. 2000. Fish as indicator for the assessment of the ecological integrity of large rivers. Hydrobiologia 422/ 423:271-278.

Thomaz, S. M.; Roberto, M. C. \& Binı, L. M. 1997. Caracterização limnógica dos ambientes aquáticos e influência dos níveis fluviométricos. In: Vazzoler, A. E. A; Agostinho, A. A. \& Hahn, N. S. eds. A planície de inundação do alto rio Paraná. Maringá, Eduem-Nupelia. p.73-102.

Vannote, R. L.; Minshall, G. W.; Cummins, K. W.; Sedell, J. R. \& Cushing, C. E. 1980. The river continuum concept. Canadian Journal of Fisheries and Aquatic Sciences 37:130-137.

Waite, I. R. \& CARPEnTER, K. D. 2000. Associations among fish assemblage structure and environmental variables in Willamette basin streams, Oregon. Transactions of the American Fisheries Society 129:754-770.

Whitton, B. A. 1975. River ecology. Berkeley, University of California. 725 p.

Wootton, R. J. 1990. Ecology of Teleost Fishes. London, Chapman \& Hall. 404 p.

1992. Fish ecology. New York, Chapman \& Hall. 212 p.

$\bar{Z}_{\mathrm{A}}$, J. H. 1999. Biostatistical Analysis. New Jersey, PrenticeHall. 663 p. 